\title{
A low power Wireless Sensor Node with Vibration Sensing and Energy Harvesting capability
}

\author{
M. Zieliński, F. Mieyeville, D. Navarro \\ Ecole Centrale de Lyon \\ Université de Lyon, \\ Institut des Nanotechnologies de Lyon \\ Ecully, F-69134, France \\ Email: mateusz.zielinski@ec-lyon.fr
}

\author{
O. Bareille \\ Ecole Centrale de Lyon \\ Université de Lyon, \\ Laboratoire de Tribologie et Dynamique des Systemes \\ Ecully, F-69134, France
}

\begin{abstract}
This paper describes the design of the wireless sensor network node (WSN) for distributed active vibration control (AVC) system for the automotive application. The approach of the system is presented in details. A WSN node using one piezoelectric element provides several features (sensing, shunting and energy harvesting). Integration of the vibration sensing capability for active vibration control system with the energy harvesting capability is described here. Simulation results are compared with the prototype design.
\end{abstract}

\section{INTRODUCTION}

W IRELESS Sensor Networks (WSNs) are made up of intelligent and autonomous nodes. Each node is able to work independently in distributed area, and is an energy aware and a low-power device. Nodes are able to establish autonomously an efficient wireless connection, which is used to send measured values. WSNs are commonly used to monitor the physical or environmental conditions like temperature, sound and vibration [1].

WSN nodes are commonly supplied from batteries. It is the last "wire" which should be cut, to provide long lifetime and maintenance-free systems. Nowadays, batteries need to be charged or replaced [2]. It introduces additional costs in the use of WSNs. Despite this inconvenience WSNs are applied in Structural Health Monitoring systems (SHMs), Environmental Monitoring systems which provide new features compared to existing wired solutions [3].

Active Vibration Control (AVC) systems become a very important issue in many engineering fields (SHM, automotive and industrial applications) which provide smart solutions for vibration and noise damping systems. Several solutions for AVC have been proposed and tested with promising results [4]. However those systems are centralised, wired and need a lot of computing power. Implementation of the AVC in a WSN is a challenge for designers. The entire vibration control system must be designed in order to pass very stringent requirements of the WSN and control law [5].

\section{RESEARCH Assumptions}

The usage of the active vibration control can reduce the weight of conventional passive methods, helping to push towards lighter, more fuel efficient vehicles [6]. Active methods include vibration sources which are driven by the control strategy algorithm to provide destructive interference of real vibrations. There are two possibilities to implement the control strategy: feed-forward (open-loop) and feedback (closedloop). A variety of algorithms have been used to adapt the controller, most are based on adaptative filtering methods: least mean square (LMS) and filtered reference LMS (FxLMS) [7]. Conventional AVC systems based on wired networks ( sensors and actuators) with high-power controller and fast data processing need a lot of energy. Replacing a big centralised (wired) system with a low power nodes can improve the AVC in the scope of functionality, maintenance costs and energy consumption.

\section{SYSTEM APPROACH}

WSNs have rather low transmission rates. Due to delays, an implementation of the real-time system which is necessary to provide data processing for centralised AVC systems is not possible [5]. In spite of, WSNs could be used to provide active control. A distributed approach used in place of the centralised one can be a solution. In our approach, intelligent nodes provide local action, which reduces the amount of the information to transfer, compared to the centralised approach. The feasibility of the distributed autonomous nodes with sensing feature coupled to semi-active vibration control dissipation is the aim of the work.

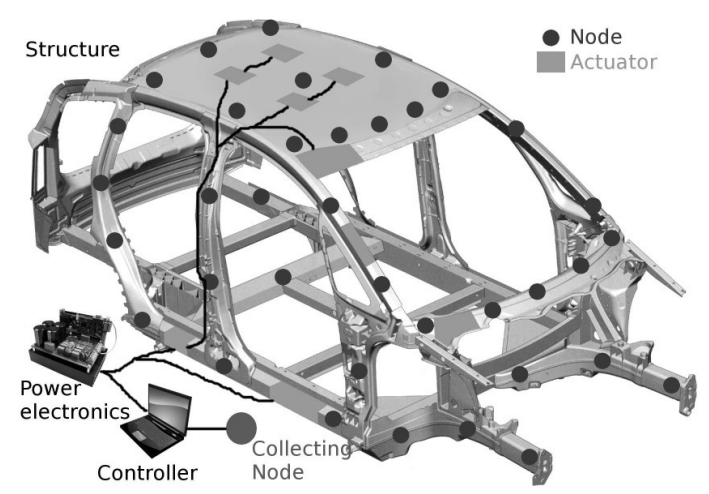

Fig. 1. System structure 
Figure 1 presents the approach of the global system structure for distributed AVC system for automotive application. Wireless nodes are implemented in the body of the car. Nodes are in the star topology towards the collecting node. Each node in the system is powered from the harvested energy and provides mechanical damping. It is a first level of the AVC system. Nodes measure the value of vibrations and if necessary send data to the collecting node, which is responsible for second level of the AVC system (high power, wired actuators). If there are no vibrations in the system, the nodes do not have energy to work but there is no need to cancel the vibrations. When vibrations occur, the WSN nodes are initiated and provide first level of the mechanical damping. If necessary they send measurements to the collecting node, and ask for a second level of the AVC.

\section{WSN NODE DESIGN ASSUMPTIONS}

The WSN node provides several hardware features: sensing, energy harvesting and local damping using one piezoelectric element. The following paragraphs describe these features and provide description of the chosen solutions.

\section{A. Sensing vibrations}

Sensing vibrations requires understanding two issues. The first one is the environment of vibrations; the second is a piezoelectric effect.

Various vibration noises have been already investigated in literature [7]. Body car vibrations are low frequency signals. Noises inside a car under operating conditions have low frequencies (less than $300 \mathrm{~Hz}$ ) and are mostly determined by acoustic resonances and body vibrations modes [8], [9].

The piezoelectric element can be used to track mechanical vibrations [10]. The output voltage of the piezoelectric element corresponds to the mechanical acceleration. It makes the piezoelectric element proper for sensing vibrations.

\section{B. Energy Harvesting}

The piezoelectric element can be described as a current source with an inbuilt capacitance. The existence of this capacitance suggests using the shunt inductor to achieve maximum power flow. However, the large value of the inductor, does not allow to use this solution in the real design. Several solutions for harvesting energy from the piezoelectric element have been already proposed. So-called "synchronised switch damping" (SSD) are semi-passive methods developed to address the problem of vibration damping. Techniques based on SSD method provide efficient energy harvesting by increasing the energy flow between the piezoelectric element and load [10].

\section{Mechanical damping}

Shunting the piezoelectric element (provided by the energy harvesting method) can be used for structural damping. The efficiency of dissipation energy in the shunt connected to the piezoelectric element is currently an issue of research. We distinguish passive and active methods. Passive methods use passive electrical elements to provide mechanical damping [11] whereas active methods use non-linear circuits like negative-capacitance [12] or switching methods [13].

\section{Chosen solutions}

The design of an autonomous and intelligent WSN node must consider requirements mentioned in previous paragraphs. Low frequency vibrations in the body of the car suggest the use of efficient energy but slow and low-computing, 8bit lowpower microcontroller with internal analog-to-digital converter (ADC). It provides low power consumption. Moreover, the sensing vibrations capability needs the piezoelectric element in the open circuit.

The choice of the energy harvesting method requires to take under consideration several parameters: efficiency of the energy harvesting, capability of vibrations damping, efficiency in the power consumption, facility of implementation and sensing capability. The series switching over the inductance (Series SSHI) method is chosen for the design. It is semipassive technique, based on non-linear processing on the piezoelectric voltage.

\section{WSN NODE DESIGN}

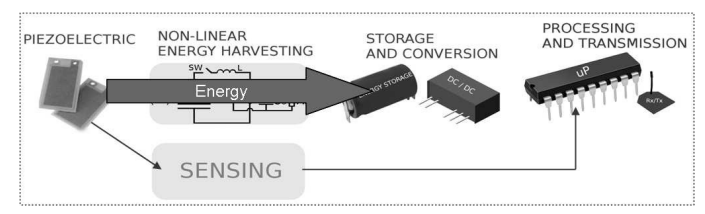

Fig. 2. WSN node schema

Figure 2 presents the schema block for WSN node. We distinguish two parallel systems. The first one for energy harvesting and damping vibrations, the second one for sensing vibrations. Both are connected to one piezoelectric patch transducer. Harvested energy is kept in the storage and used to supply the microprocessor and wireless transmission. The following paragraphs describe the design of the WSN node.

\section{A. Piezoelectric element}

Piezoelectric effect can be considered as a bidirectional energy conversion. A strain on the piezoelectric element generates the electrical tension and respectively the electrical tension over the piezoelectric element generates the mechanical strain.

To understand electrical properties of the piezoelectric patch transducer several measurements have been done. Figure 3 and figure 4 presents achieved results.

The current and voltage values are measured over the piezoelectric patch transducer in function of the resistive load for constant frequency (figure 3). The piezoelectric element is a real current source and for the optimal resistive load provides the maximal power (figure 4). It clearly shows that the energy harvesting circuit must be designed in accordance with the electrical properties of the piezoelectric patch (optimal load).

According to figures 3 and 4 we can observe that the high value of the resistive load reduces the amount of the energy 


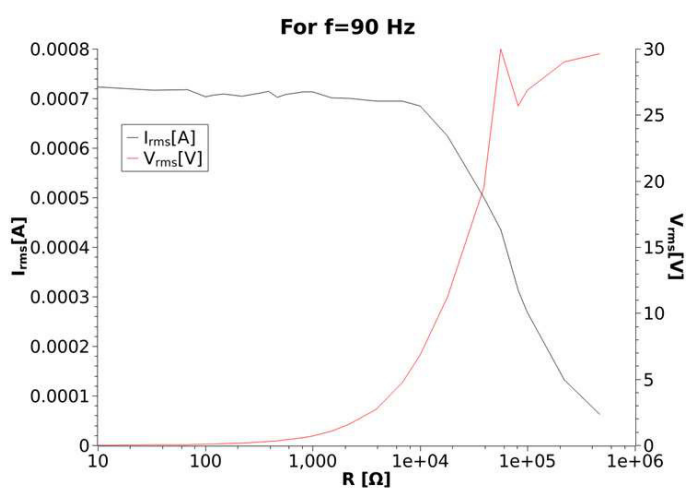

Fig. 3. Output electrical characteristics for piezoelectric patch transducer

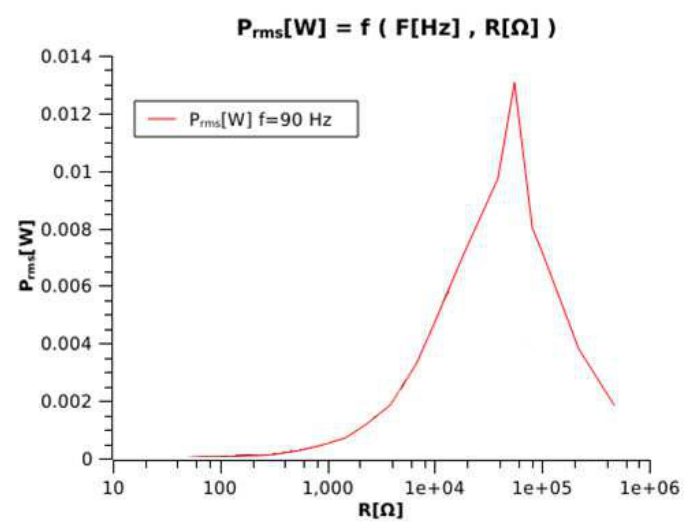

Fig. 4. Power received from the piezoelectric patch transducer in function of resistive load

received from the piezoelectric element. It proves the usage of the piezoelectric element with high resistive load for sensing. This solution provides a good resolution of measurements due to high voltage values and low energy leakage.

\section{B. Sensing vibrations}

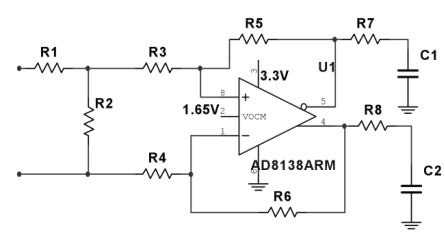

Fig. 5. Sensing circuit

The designed circuit for sensing vibrations and signal conditioning is presented on figure 5. It is composed of the voltage divider (R1 and R2) and the AD8138 low distortion differential analog-to-digital (ADC) driver from Analog Devices. The low pass filter is used to cut-off high frequencies over the output of the ADC driver ( $\mathrm{R} 7$ with $\mathrm{C} 1$ and $\mathrm{R} 8$ with $\mathrm{C} 2$ ). The differential ADC driver provides also offset voltage (Pin 2 connected to the $1.65 \mathrm{~V}$ ). Hence, the negative and positive values are measured, it is necessary to provide active vibration control. In the designed circuit the piezoelectric element is not connected directly to the circuit ground. The low-power differential amplifier is supplied from single $3.3 \mathrm{~V}$, which simplifies the supply circuit. An internal ADC of the microcontroller is used. This solution provides low energy consumption since there is no additional ADC to supply.

\section{Switching circuit for SSHI method}

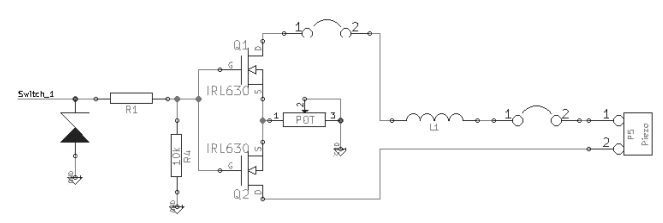

Fig. 6. Switching circuit for SSHI method

The series SSHI circuit is presented on the figure 6. It contains two IRL630 NMOS transistors driven by the microcontroller (the full-wave switch circuit with low current leakage and low short-circuit resistance). The usage of the logic-level transistor simplifies the circuit; the output of the microcontroller can be used to drive the switch. The zener diode D1 is used to protect the microcontroller pin, the R1 resistor sets the current value (it is correlated with the turn-on time). The R4 is used to reduce the turn-off time.

In the series SSHI method, the inbuilt piezoelectric capacitance and external inductance creates the series resonant circuit. The switch keep the circuit in the open-circuit. While the extremum of the mechanical displacement is detected, the switch is closed for half of the electrical resonant period. It causes inversion of the piezoelectric element voltage. The period of the mechanical displacement is much longer than the period of the electrical circuit.

\section{Vi. Simulations}

Designed circuits: vibration sensing and energy harvesting are simulated in the NI Multisim Component Evaluator 13.0 from National Instruments [14]. Devices used in the design are implemented using SPICE models. The sensing circuit and the series SSHI switching circuit are connected in parallel with the model of the piezoelectric element.

Figure 7 a shows simulated signal for sensing vibrations. The $1.65 \mathrm{~V}$ offset is used for signal conditioning, hence positive and negative parts of the signal can be measured in the ADC of the microcontroller.

Figure $7 \mathrm{~b}$ shows simulation results for switching circuit while the switch is open. The sinusoidal signal is a voltage over the piezoelectric element. The second wave - small peaks represents the current in the circuit.

Figure $7 \mathrm{c}$ shows simulation results for switching circuit while the switch is closed. The sinusoidal signals presented on figure correspond to the voltage and current in the circuit. The shift phase between them proves existence of the capacity (inbuilt capacity of the piezoelectric element). 


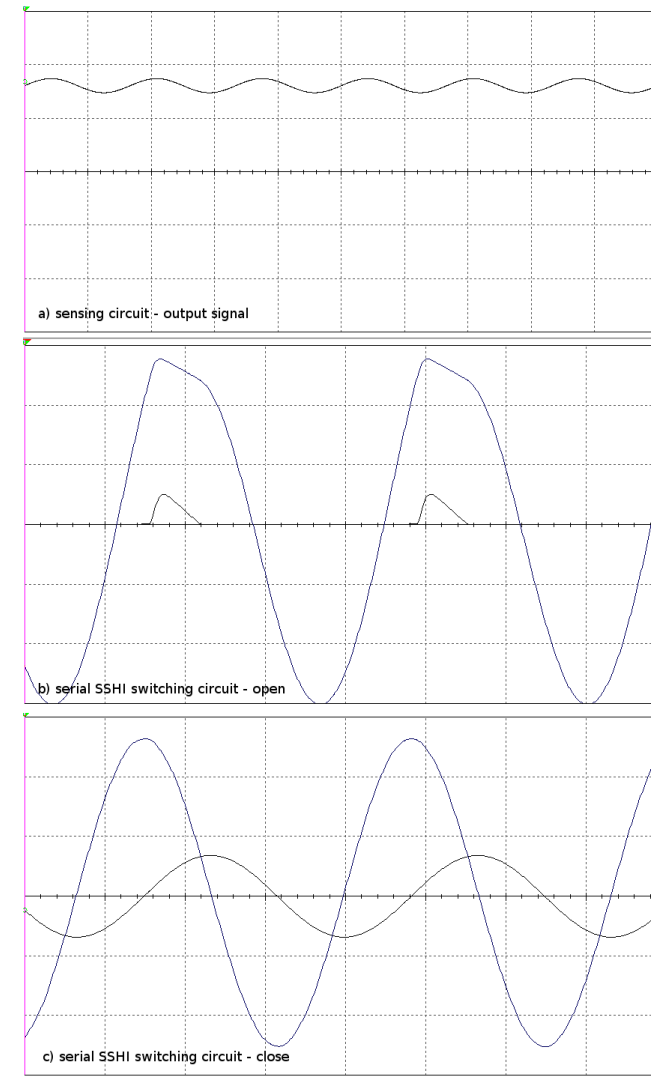

Fig. 7. Simulation results

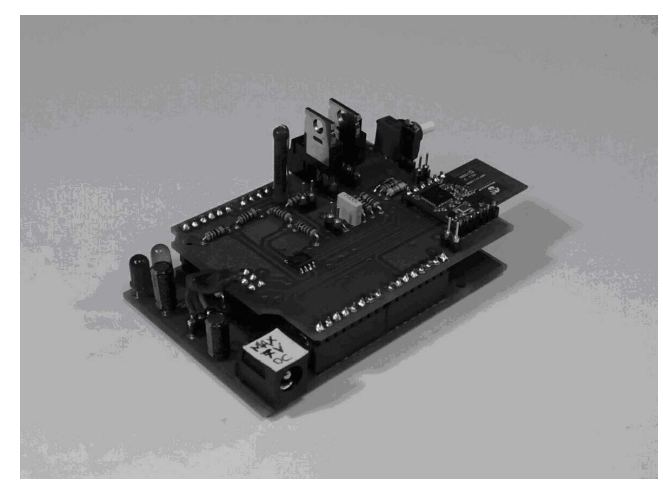

Fig. 8. Photo of the prototype WSN node

\section{IMPLEMENTATION}

Figure 8 presents the photo of the real prototype device. It is composed of two PCB boards. The first board contains microcontroller and supply circuit, the second provides the SSHI circuit, sensing circuit and wireless communication. In designed prototype series SSHI circuit and sensing circuit are connected in parallel with the piezoelectric patch transducer which corresponds to the simulations.

For wireless communication we used the MRF24J40 radiofrequency transceiver from Microchip. It provides hardware support for IEEE 802.15.4 physical layer. The node is equipped with low-power microcontroller PIC16LF88 with internal 10bit ADC.

\section{A. Measurement station}

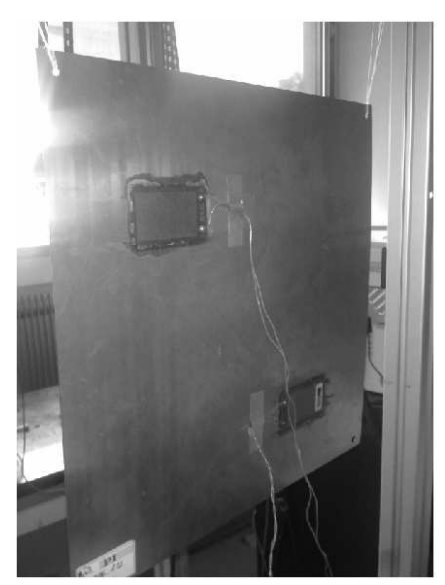

Fig. 9. Measurement station: metal plate with two piezoelectric patches

The measurement station used for tests is presented on figure 9. It contains two PI-876 piezoelectric patch transducers from PI Ceramic [14]. Vibrations are generated by an attached electric vibrator which is connected with the signal amplifier and function generator. Acceleration is measured by the external accelerometer connected directly to the plate next to the piezoelectric element (accelerometer sensitivity 10,43 mV/g).

\section{B. Validation of sensing circuit}

Figure 10 presents the validation of the sensing circuit. The WSN node is supplied; the transistor-switch is open (sensing capability). The first channel $(\mathrm{CH} 1)$ presents the voltage over the ADC (with $1.65 \mathrm{~V}$ offset). It corresponds to simulations (figure $7 \mathrm{a}$ )

\section{Validation of the switching circuit}

Figure 11 presents waveforms of voltage and current while the transistor-switch is open. The value of the current is measured over the small series resistance added to the switching circuit. The $\mathrm{CH} 1$ channel presents the piezoelectric voltage, while the $\mathrm{CH} 2$ channel presents the current in the switching circuit. The values obtained from measurements are in keeping with its simulated counterparts. (figure $7 b$ )

Figure 12 presents waveforms while the transistor-switch is closed. In this case the $\mathrm{CH} 1$ channel presents the acceleration waveform; while $\mathrm{CH} 2$ presents the current in the circuit. Figure 12 also presents the phase shift between voltage and current waveform (the capacitance character of the piezoelectric element). Obtained measured values correspond to simulations (figure 7c)

\section{DISCUSSION, PERSPECTIVES}

The sensing circuit and switching circuit have been designed separately to provide the best solution for each one. Both circuits have been simulated and implemented in the prototype 


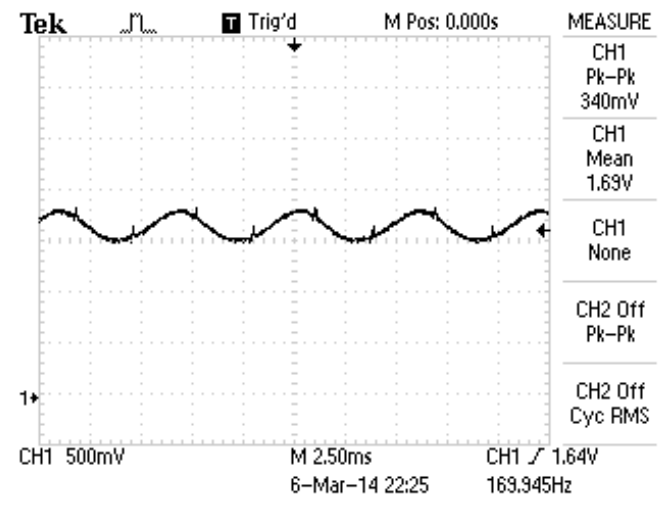

Fig. 10. Validation of the sensing circuit

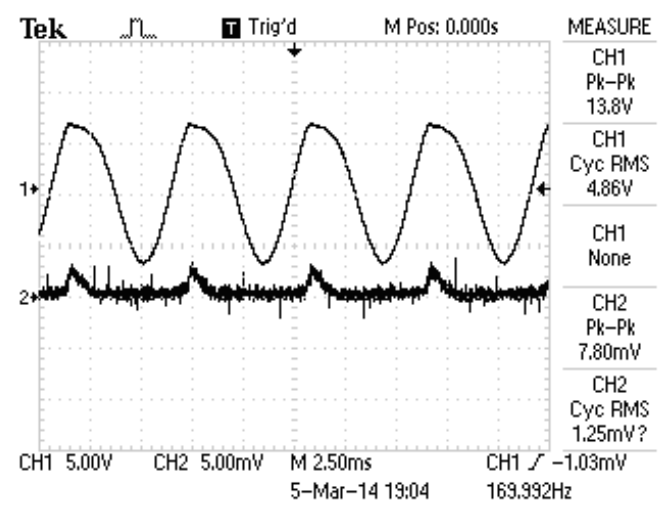

Fig. 11. Transistor-switch open

design. Achieved measurements correspond to the simulations. According to figures $7 \mathrm{~b}, 11,7 \mathrm{c}$ and 12 the relative error between simulations and real measurements is $9.2 \%$.

The $\mathrm{CH} 2$ trace on figure 11 presents current in the switching circuit while the switch is open. It is default current caused by lack of the insulation between ground referenced switch, floating-source piezoelectric generator and differential-mode sensing circuit.

To provide galvanic separation (switch circuit not referenced to the ground) two solutions are considered: optical isolation [16], [18] and transformer [17], [18].

The optical isolation is recommended for its reliability and simple implementation. The disadvantage of this method is requirement of the second separated supply circuit to drive the MOSFET switch. It would add additional devices in the supply circuit. Since the WSN node is designed in order to verify the self-supply capability, the use of the additional devices which introduce energy losses should be avoided.

The second approach is to use the transformer to drive NMOS transistors. A pulse transformer is, in principle, a simple, reliable and highly noise-immune method of providing isolated gate drive. It can be advised for applications were the duty cycle is small. As a stand-alone component it can be used for duty cycles between $35 \%$ and $65 \%$ [19].

The series SSHI is a system with low duty cycle because

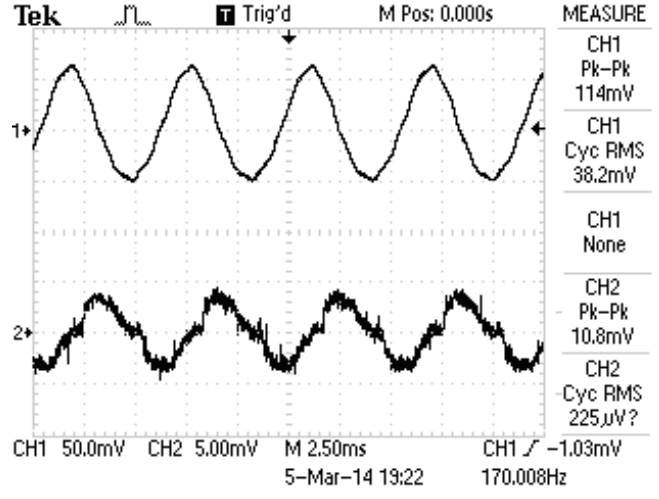

Fig. 12. Transistor-switch closed

the period of the mechanical displacement is much longer than period of the electrical circuit. It makes the pulse transformer a promising solution for our design.

Simulations have been realised to validate the circuit with the chosen solution (pulse transformer). The NI Multisim Component Evaluator 13.0 from National Instruments is used. The sensing vibrations circuit and the series SSHI circuit are in parallel with the piezoelectric element (design contains a switch not referenced to the ground). The entire simulation schema is presented on figure 13. The individual parts of the design are marked on the figure. We distinguish the switch driver composed of the voltage source V1 (corresponds to the microcontroller output signal in the real design). The series SSHI circuit contains two transistors and the inductor L2. Additionally, the bridge circuit (diodes: D1, D2, D3 and D4) is used to transform voltage from AC to DC. Finally, the load is simulated by the resistor $\mathrm{R} 1$ and the capacitor $\mathrm{C} 1$. The sensing circuit is connected in parallel with the SSHI circuit. Figure 14 compares the theoretical waveforms with the simulation results of the series SSHI method. Simulation results correspond to the theoretical waveforms but RLC and diode impacts are visible. The results justify the correctness of the integration of the sensing circuit with the energy harvesting circuit.

\section{CONCLUSION}

Big centralised and wired systems for active vibration control are costly and use a large quantity of energy. A distributed solution based on an energy aware wireless sensor network has been proposed as a replacement for the centralised system. The autonomous WSN node needs to be designed to provide efficient wireless network for distributed active vibration control.

In this paper the global approach and the system assumptions are established and used as input data for the design. The proposed design of the WSN node is in accord with the prescribed requirements.

Designed node provides: vibration sensing, shunting the piezoelectric element and wireless communication. Furthermore, the series SSHI technique, chosen for the design, provides damping of the mechanical vibrations and the energy harvesting capability. 


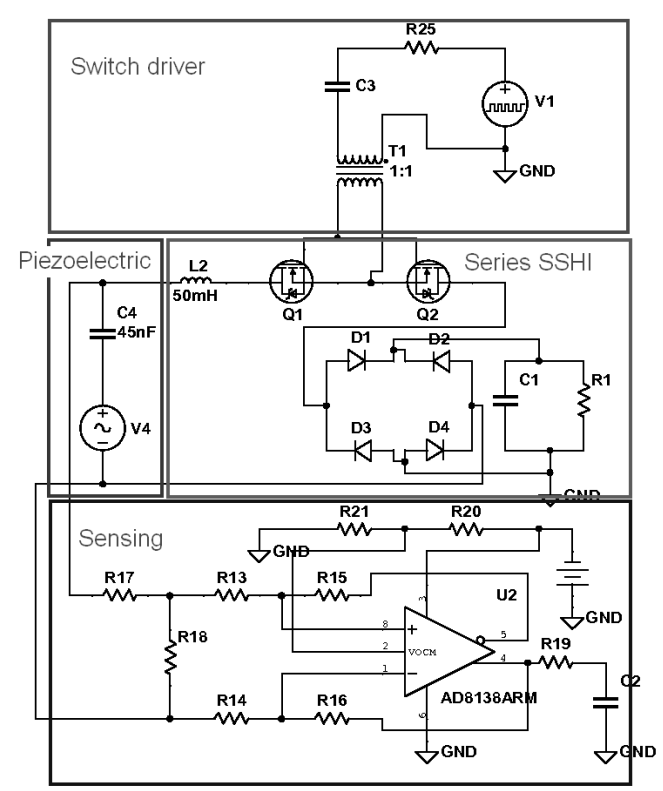

Fig. 13. Simulated circuit

Designed circuits for sensing vibrations and shunting the piezoelectric element are presented and described in details. The WSN node is modelled using the SPICE models. Achieved simulation results are consistent with the expected ones and validate the design.

The WSN node prototype has been constructed. The simulation results are compared with the measurements. The metal plate with the attached piezoelectric element is used as a measurement station. The measurement results correspond to the simulation results. The correctness of the simulations is proved with the measurements (relative error is $9.2 \%$ ).

Finally the separation problem for self-powered circuits has been indicated. Possible solutions are taken under consideration. The circuit with the chosen solution (pulse transformer) is simulated; obtained results correspond to the theoretical research (expected results).

The next step of the work would be modification of the prototype device according to the presented simulations. The galvanic separation for non-ground referenced switch will be used. The measurements would be compared with the simulation results. The following step would be validation of the energy harvesting and mechanical damping capability.

\section{REFERENCES}

[1] J. Yick, B. Mukherjee, D. Ghosal. August 2008. "Wireless sensor network survey" Computer Networks vol. 52 p.2292-2330, http://dx.doi.org/10.1016/j.comnet.2008.04.002

[2] J. Micek, J. Kapitulik. September 2012. "WSN sensor node for protected area monitoring" Federated Conference on Computer Science and Information Systems p.803-807, https://fedcsis.org/proceedings/2012/pliks/237.pdf

[3] J. P. Lynch. 2007. "An overview of wireless structural health monitoring for civil structures" Philosophical Transactions, Royal Society A, p. 345372, http://dx.doi.org/10.1098/rsta.2006.1932
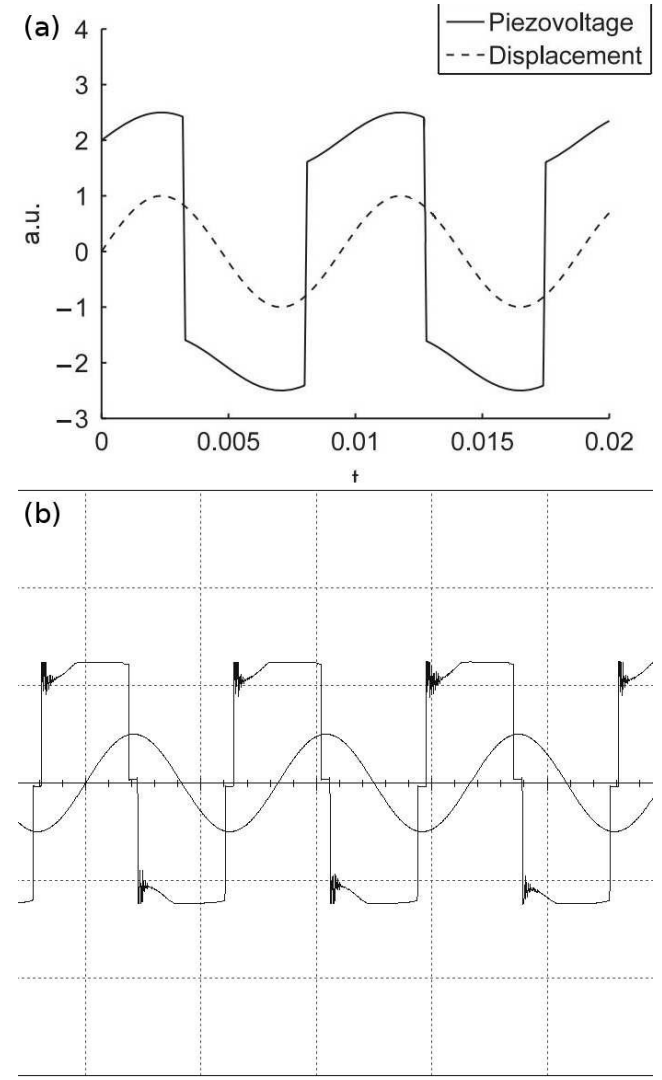

Fig. 14. (a) theoretical SSHI waveforms [10] (b) simulated SSHI waveforms

[4] F. Svaricek, T. Fueger, H. Karkosh, P. Marienfeld, C. Bohn. September 2010, Sciyo, Croatia. "Automotive Applications of Active Control", ISBN 978-953-307-117-6, pp. 380, http://cdn.intechopen.com/pdfswm/11899.pdf

[5] F. Mieyeville, M. Ichchou, G. Scorletti, D. Navarro, W. Du. 2012 "Wireless Sensor networks for active vibration control in automobile structures". Smart Mater. Struct 21, http://dx.doi.org/10.1088/09641726/21/7/075009

[6] S.J. Elliott. December 2008. "A review of Active Noise and Vibration Control in road vehicles", ISVR Tehcnical Memorandum No 981, http://eprints.soton.ac.uk/id/eprint/65371

[7] C.R. Fuller, A.H. Von Flotow. December 1995. "Active Control of Sound and Vibration" IEEE Control System, http://dx.doi.org/10.1109/37.476383

[8] L. Hermans and H. Van Der Auweraer. 1999. "Modal testing and analysis of structures under operational conditions: industrial applications" Mechanical Systems and Signal Processing 13(2), 193-216, http://dx.doi.org/10.1006/mssp.1998.1211

[9] S. H. Kim, J. M. Lee, M. H.Sung. 1999. "Structural-Acustic Modal Coupling Analysis and Application to Noise Reduction in a Vehicle Passenger Compartment". Journal of Sound and Vibration 255(5), p. 989-999, http://dx.doi.org/10.1006/jsvi.1999.2217

[10] E. Lefeuvre, A. Badel, C. Richard, L. Petit, D. Guyomar. 2006. "A comparison between several vibration-powered piezoelectric generators for standalone systems" Elsevier, Sensors and Actuators A 126 p. 405416, http://dx.doi.org/10.1016/j.sna.2005.10.043

[11] Jin-Young Jeon. 2009. "Passive vibratin damping enhancement of piezoelectric shunt damping system using optimization approach", Journal of Mechanical Science and Technology, 23, http://dx.doi.org/10.1007/s12206-009-0402-8

[12] B de Marneffe, A Preumont. 2008. "Vibration damping with negative capacitance shunts: theory and experiment", Smart Materials and Structures 17, http://dx.doi.org/10.1088/0964-1726/17/3/035015

[13] Saber Mohammadi, Akram Khodayari. 2012. "Damping analyses of 
structural vibratins and shunted piezoelectric transducers", Smart Materials Research, http://dx.doi.org/10.1155/2012/431790

[14] NI Multisim Component Evaluator White Paper. May 2012. Internet: http://www.ni.com/white-paper/9452/en/

[15] PI Piezo Technology, P-876 DuraAct Patch Transducer White Paper. 2014. Internet: http://piceramic.com/product-detail-page/p-876101790.html

[16] E. L. Worthington, M. Zhu, P. Kirby. 2010. "Piezoelectric Energy Harvesting: Enhancing Power Output by Device Optimisation and Circuit Techniques". $\mathrm{PhD}$ Thesis, Cranfield University, https://dspace.lib.cranfield.ac.uk/

[17] L. Balogh. "Design And Application Guide for High Speed MOSFET Gate Drive Circuits", Texas Instruments, http://www.ti.com/lit/ml/slup169/slup169.pdf

[18] Fairchild Semicunductor. 2007. "Application Note AN-6069; Application review and comparative evaluation of low-side gate drivers", http://www.fairchildsemi.com/an/AN/AN-6069.pdf

[19] Vishay Siliconix. 2010. "Application Note AN-937;Gate Drive Characteristics and Requirements for HEXFET Power MOSFETs". Document Number: 91421, http://www.vishay.com/docs/91421/appnote9.pdf 